ORIGINAL ARTICLE

\title{
Cardiopulmonary resuscitation on Flemish television: challenges to the television effects hypothesis
}

\author{
J Van den Bulck, K Damiaans
}

Emerg Med J 2004;21:565-567. doi: 10.1136/emj.2003.010819

See end of article for authors' affiliations

......................

Correspondence to: Professsor J Van den Bulck Katholieke Universiteit Leuven, Department of Communication, Van Evenstraat 2 A, 3000 Leuven, Belgium; jan.vandenbulck@soc. kuleuven.ac.be

Accepted for publication 31 October 2003

\begin{abstract}
Background: People who watch a lot of medical fiction overestimate the success rate of cardiopulmonary resuscitation (CPR). It has been suggested that this is because CPR is usually shown to be successful on television. This study analysed a popular Flemish medical drama series. Previous research showed that heavy viewing of this series was related to overestimation of CPR success.

Method: Content analysis of 70 episodes of "Spoed" in the period between 2001 and the first three months of 2003. Causes and treatment of cardiac arrest and outcome of CPR were recorded in the same way as previous studies.

Results: CPR was performed 31 times in the 70 episodes. Only 19\% of the patients survived the resuscitation attempt. Most patients were middle aged or older. Causes of arrest were different from those in British or American television series.

Conclusions: The low survival rate challenges the idea that heavy viewers adopt the overestimation shown by television. Psychological research shows that people ignore base rate information in the shape of statistics, in favour of vivid, dramatic examples. Showing some impressive examples of success might therefore be more important than the overall success rate. It is suggested that the message of television fiction is that doctors are not powerless and that treatment does not stop once the heart stops beating. This helps to create what has been called an "illusion of efficacy".
\end{abstract}

n 1996 Diem and colleagues were the first to study the way in which cardiopulmonary resuscitation (CPR) is portrayed on fictional television. ${ }^{1}$ The study looked at 97 episodes of medical television fiction shows and found that $75 \%$ of the patients survived resuscitation after cardiac arrest, that most arrests were the result of trauma, and that most occurred in young people. As the authors note, this is exactly the opposite of what happens in real life. Subsequently, a British study looked at 64 episodes of a British medical series. ${ }^{2}$ On British television patients were young, too, but the outcome of the resuscitation attempts was closer to the real world statistic.

Both studies assume that television drama has an effect on the perceptions and attitudes of viewers regarding the outcome of CPR. This hypothesis seems to be supported by two studies. One study of 269 church goers in the United States found that people who indicated that television was their main source of information regarding CPR, gave higher estimates of surviving cardiac arrest after administration of CPR. ${ }^{3}$ A Flemish study of 820 secondary school children showed that children who regularly watched a popular, locally produced, emergency room drama series (called "Spoed") gave statistically higher estimates of CPR survival rates, compared with children who did not watch the series. ${ }^{4}$ The author suggests that this is a "first order cultivation effect" of television, a concept used by media effects researchers to refer to the process whereby heavy television viewers start to adopt "assumptions about the facts of life that reflect the medium's most recurrent portrayals". ${ }^{5}$ Both the American and the Flemish effects study assume that watching medical television drama in which resuscitation attempts are nearly always successful are what induces a television viewer to ascribe higher success rates to in-hospital CPR. Neither study shows a causal link because both are based on cross sectional survey methods. The American study is backed up by the study by Diem et al suggesting that in recent American medical drama the success of CPR is overestimated. The British content analysis shows, however, that overestimating the efficacy of CPR is not a universal feature of television drama. It may, therefore, be typical of American television drama only.

This study looks at the presentation and prevalence of CPR in the television series to which the Flemish study referred. If the content analysis finds an overestimation of the effectiveness of CPR, as was the case in the American studies, the "first order cultivation hypothesis" of the previous effects studies receives additional support. If, however, the Flemish series more closely resembles the British examples and downplays the effectiveness of CPR, the media effects model is challenged and another explanation for the relation between television viewing and overestimating the success rate of CPR will be required.

\section{METHODS}

We viewed 70 episodes of "Spoed" during 2001 to the beginning of 2003. "Spoed" means "urgency" or "emergency", it is the word used in the name for emergency rooms in both Flanders (the Dutch speaking region of Belgium) and the Netherlands. Spoed is the most popular medical drama series in Flanders, with a viewer share that regularly exceeds $35 \%$ of the viewing audience. The show is also broadcast in the Netherlands.

For purposes of comparison and replication we used the same definition of CPR as was used by Diem and colleagues ${ }^{1}$ and also used the same variables for analysing the cases of $\mathrm{CPR}$ in the series.

A datasheet was compiled for each instance where some form of CPR was performed.

\section{RESULTS}

Diem et al found 60 occurrences of CPR in 97 episodes, ${ }^{1}$ Gordon et al found 52 cases in 60 episodes. ${ }^{2}$ In our study 31 cases of CPR were registered in 70 episodes. This represented $43 \%$ of all cases of cardiac arrest (total number of cardiac arrests was 58). 
Overall, $81 \%$ of the patients did not survive the resuscitation attempt. Six per cent survived to the point of hospital discharge and 13\% survived, but information regarding hospital discharge was not provided. In total, therefore, the series only showed a $19 \%$ success rate of in-hospital CPR attempts.

Most patients suffering cardiac arrest were (or appeared to be) middle aged or older (see table 1).

Causes of cardiac arrest as shown or suggested by the series were very different from those in the American or the British examples. Only $43.3 \%$ of the causes fell in the categories used by Diem et al and only $69 \%$ fell into the categories of Gordon et al (see table 2).

In $6.4 \%$ of the cardiac arrests no attempt was made to reestablish ventilation of the patient in any way. In another $6.4 \%$ of cases it was impossible to see what was being done regarding ventilation. Chest compressions were used in $71 \%$ of the cases. Frequency of the compressions was between 80 to 100 per minute in $32 \%$ of the cases. It was slower than that in $41 \%$ of cases and faster in $23 \%$. Frequency could not be ascertained in $4 \%$ of the cases. In $58 \%$ of the cases some form of defibrillation was applied.

\section{DISCUSSION}

The content analysis of the Flemish television series mirrors the findings of the British study by Gordon et al in that CPR was usually unsuccessful. However, cardiac arrest appeared to occur in older rather than younger people. The overall success rate of $19 \%$ is therefore closer to the real world answer and very different from the findings regarding medical series produced in the United States.

These findings challenge the idea that perceptions of CPR are influenced by the way it is presented in television fiction. An association between watching a lot of television and overestimation of the effectiveness of CPR was found in the United States, but a Flemish study found that people who watch "Spoed" regularly also overestimated the effectiveness of CPR. On average they estimated that $62.4 \%$ of patients survived in-hospital CPR. The content analysis presented in this article challenges the hypothesis that what occurred in the Flemish case is an example of a first order cultivation effect, because that effect occurs when people adopt the picture of the world presented in television fiction. An alternative explanation must be sought.

Cognitive psychology and media effects research might offer such an alternative explanation. Firstly, it has to be remarked that television viewers do not (re-)produce statistics like those presented in this article while they are viewing. It has been shown that people do not form such judgments about the prevalence of events in the real world while they are watching television. If there is an effect of television, it is more likely to occur when people are asked to offer or form a judgment, ${ }^{6}$ for example, when answering a researcher's questions or when making a decision requiring an idea of the success rate of CPR (as would be the case when "do not resuscitate" decisions have to be taken). Secondly, social cognition theory shows that people have a tendency to
Table 2 Causes of cardiac arrest in a Flemish television series

\begin{tabular}{lll}
\hline Cause & $\begin{array}{l}\text { Frequency } \\
\text { (n=31) }\end{array}$ & Percentage \\
\hline Gunshot wound & 5 & 16.1 \\
Assault (including stabbing) & 6 & 19.4 \\
Cardiac & 5 & 16.1 \\
Explosion/fire & 3 & 9.7 \\
Overdose & 2 & 6.5 \\
Motor vehicle accident & 2 & 6.5 \\
Other trauma & 2 & 6.5 \\
Suicide & 1 & 3.2 \\
Self inflicted cuts (accidental) & 1 & 3.2 \\
Hypothermia & 1 & 3.2 \\
Surgical error & 1 & 3.2 \\
Poison gas & 1 & 3.2 \\
Pneumonic plague & 1 & 3.2 \\
\hline
\end{tabular}

"ignore general, broadly based information about population characteristics (that is, base rate information) in favour of more concrete anecdotal but usually less valid and reliable information". ${ }^{7}$ In other words, a number of dramatic rescues entailing CPR might have a larger effect on the viewer's perceptions than a much larger number of unsuccessful attempts. Diem et al remarked that the intervention of medical resuscitation experts is often presented as a "miracle", ${ }^{1}$ while even failed attempts are usually presented in the context of "the doctors did all they could to save the patient". In that sense even failed attempts are an example of the power of medical intervention, not of its failure.

\section{CONCLUSIONS}

The positive and negative implications of overestimating the success of CPR as a result of television's portrayal of it have been discussed at length elsewhere. ${ }^{1-4}$ This article suggests that the case of "Spoed" sheds a different light on these previous studies regarding the impact of television on perceptions of CPR. We argue that the message of television to the lay audience is not whether or not CPR is a technique that has a high or low success rate, but rather that cardiac or respiratory arrest, or both, need not be the end of one's life and that physicians and other trained responders can perform procedures and protocols that can sometimes reverse the arrest. The distinction between successful and nonsuccessful CPR might be a false one in the eyes of the public. The real distinction is between, on the one hand, the perception that when the heart stops beating and the person stops breathing nothing else can be done and, on the other hand, the perception that medical experts are not yet at the end of their powers when such an event occurs. This might explain why a similar "illusion of efficacy" occurs in trained medical personnel ${ }^{8}$ and among the Flemish respondents who had undergone CPR training. ${ }^{4}$ These people have learned (at various levels of mastery and sophistication) that cardiac arrest need not be irreversible. They appear to experience the

Table 1 Age groups of patients suffering cardiac arrest in a Flemish television series

\begin{tabular}{llllll}
\hline \multirow{2}{*}{ Age } & \multicolumn{2}{l}{ CPR attempted $(\mathbf{n}=\mathbf{3 1})$} & & \multicolumn{2}{l}{ No CPR attempted $(\mathbf{n}=33)$} \\
\cline { 2 - 3 } & Frequency & Percentage & & Frequency & Percentage \\
\hline Children & 4 & 13.0 & 4 & 12.0 \\
Teenagers & 1 & 3.2 & 6 & 18.0 \\
Young adults & 8 & 25.8 & & 9 & 27.0 \\
Middle aged people & 9 & 29.0 & & 13 & 39.0 \\
Senior citizens & 9 & 29.0 & & 1 & 3.0 \\
\hline
\end{tabular}


same, inescapable, predisposition to ignore base rate information regarding the success rate of CPR in favour of the more vivid perception that something can be done, even when the heart has stopped beating.

\section{Authors' affiliations}

J Van den Bulck, K Damiaans, Katholieke Universiteit Leuven,

Department of Communication, Leuven, Belgium

Funding: none.

Conflicts of interest: none declared.

\section{REFERENCES}

1 Diem SJ, Lantos JD, Tulsky JA. Cardiopulmonary resuscitation on television: miracles and misinformation. N Engl J Med 1996;334:1578-82.
2 Gordon PN, Williamson S, Lawler PG. As seen on TV: observational study of cardiopulmonary resuscitation in British television medical dramas. BMJ 1998;317:780-3.

3 Jones GK, Brewer KL, Garrison HG. Public expectations of survival following cardiopulmonary resuscitation. Acad Emerg Med 2000;7:48-53.

4 Van den Bulck J. The impact of television fiction on public expectations of survival following inhospital cardiopulmonary resuscitation by medical professionals. European Journal of Emergency Medicine 2002:9:325-9.

5 Morgan M, Shanahan J. Two decades of cultivation research: an appraisal and meta-analysis. Communication Yearbook 1997;20:1-45.

6 Shrum $\amalg$. Media consumption and perceptions of social reality: effects and underlying processes. In: Bryant J, Zillmann D, eds. Media effects: advances in theory and research. 2nd ed. Mahwah, NJ: Lawrence Erlbaum Associates, 2002:69-95.

7 Fiske ST, Taylor SE. Social cognition. New York: McGraw-Hill, 1991.

8 Lewis RJ. Emergency medicine's "illusion of efficacy" and the public perception of resuscitation research. Acad Emerg Med 1999;6:771-2 